\title{
Mathematical modeling and computation of composite cylindrical shells under axisymmetric loading
}

\author{
Yuriy Yakubovskiy ${ }^{1}$, Irina Donkova ${ }^{2,},{ }^{,}$Aleksandr Kuzyaev ${ }^{1}$ and Mikhail Kruglov ${ }^{1}$ \\ ${ }^{1}$ Tyumen Industrial University, Volodarskogo str., 38, Tyumen, 625000, Russia \\ ${ }^{2}$ Tyumen State University, Volodarskogo str., 6, Tyumen, 625003, Russia
}

\begin{abstract}
The paper suggests an approach of mathematical modeling of composite cylindrical shells under axisymmetric load. The stress-strain state of each layer is characterized by a system of forces, moments, strains, and displacements adopted in the classical theory using Kirchhoff-Love's hypotheses (L. I. Balabuha - I. V. Novozhilov variant). The study of the stress-strain state is accomplished in accordance with the statements and hypotheses of the complex structures deformation theory taking into account shear stresses in joints. There have been built mathematical models for composite structures in the form of a circular cylindrical shell, closed in a transverse direction and bounded by two plane sections perpendicular to the axis of the cylinder. Computation of a two-layered cylindrical hinged shell under axisymmetric load has been performed.
\end{abstract}

\section{Introduction}

Composite structures formed by layers of materials with different physical and mechanical properties are used as protective constructions in oil and gas industry complexes. The connection of individual layers is made up by continuous or discrete type finite rigidity braces. A shift of layers under loadispossible; therefore, the stress-strain state is studied in terms of multilayered structures with delamination. The above research area is related to the theory of composite structures [1,2,3] for composite rods, plates and shallow shells. The theory of complex structures deformation in terms of shearing stresses in joints is applied to composite cylindrical shells. According to the theory [1] composite shells are structures consisting of several layers with finite rigidity braces pliable in the longitudinal direction and absolutely rigid in the transverse direction.

\section{Mathematical model}

A composite cylindrical structure consisting of separate $i+1$ layers and joints is under consideration. It is assumed that deflection $w$ is the same for all the package layers, and each individual shell is regarded as $\mathrm{i}$-th layer thicknessh ${ }^{(i)}$.

"Corresponding author: irina donkova@mail.ru, i.a.donkova@utmn.ru 
The stress-strain state of each structure layer is determined by a system of forces, moments, deformations and displacements, used in the classical theory applying KirchhoffLove's hypotheses (L.I. Balabuha - I.V. Novozhilov variant). The hypotheses are not applied to the whole package; thickness metric change is not regarded [6].

To describe the structure behavior the curvilinear orthogonal right-handed coordinate system is used: $x, \varphi, z^{(i)}$. The middle surface of the $\mathrm{i}$-thshell layer is related to coordinate surface $z^{(i)}=0$. Coordinate $x$, $\varphi$ lines coincide with the middle surface main curvatures lines, axisz $^{(\mathrm{i})}$ is straight. With $\mathrm{x}$, the distance along the generatrix is $(0 \leq x \leq L), \varphi$ is the angle along directing line $(0 \leq \varphi \leq 2 \pi) z^{(i)}\left(-\frac{h^{(i)}}{2} \leq z^{(i)} \leq \frac{h^{(i)}}{2}\right) ;$ Lame parameters take the values of $A_{1}^{(i)}=1, A_{1}^{(i)}=r$. The shell thickness is measured from the middle surface in the direction of coordinate $z^{(i)}$ The distances $a^{(i)}, b^{(i)}, c^{(i)}$ from the middle surface to the joint are entered; the distance between the median surfaces is $c^{(i)}=a^{(i)}+b^{(i)}$.

In the selected coordinate system, the displacement of a point on the middle surface of each i-thlayer has components $u^{(i)}, v^{(i)}, w$ along axescomponents $x, \varphi, z^{(i)}$.

The deformation parameters of the composite cylindrical shell $i$-thlayers median surfaces in displacements will be represented as:

$$
\begin{aligned}
& \varepsilon_{1}^{(i)}=\frac{\partial u^{(i)}}{\partial x}, \varepsilon_{2}^{(i)}=\frac{1}{r}\left(\frac{\partial v^{(i)}}{\partial \varphi}+w\right), \kappa 1=-\frac{\partial^{2} w}{\partial x^{2}}, \kappa_{2}^{(i)}=\frac{1}{r^{2}}\left(\frac{\partial v^{(i)}}{\partial \varphi}-\frac{\partial^{2} w}{\partial \varphi^{2}}\right) \\
& \gamma_{12}^{(i)}=\frac{\partial v^{(i)}}{\partial x}+\frac{1}{r} \frac{\partial u^{(i)}}{\partial \varphi}, \chi_{12}^{(i)}=\frac{1}{r}\left(\frac{\partial v^{(i)}}{\partial x}-\frac{\partial^{2} w}{\partial x \partial \varphi}\right),
\end{aligned}
$$

Where $\varepsilon_{1}^{(i)}, \varepsilon_{2}^{(i)}, \gamma_{12}^{(i)}$ are tangential deformation parameters; $\kappa_{1}, \kappa_{2}^{(i)}$ are curvature change parameters; $\chi_{12}^{(i)}$ is a torsion parameter.

The values of deformations in the equidistant surfaces, located at distance $z^{(i)}$ from the i-thlayer middle surface will be represented as[3]:

$$
\varepsilon_{1(z)}^{(i)}=\varepsilon_{1}^{(i)}+{ }_{z}^{(i)} k 1 ; \varepsilon_{2(z)}^{(i)} \approx \varepsilon_{2}^{(i)}+{ }_{z}^{(i)} k_{2}^{(i)} ; \gamma_{12(z)}^{(i)}=\gamma_{12}^{(i)}+{ }_{z}^{(i)} \chi_{12}^{(i)}
$$

Generalized Hooke's law is used as physical correlations for a structure material considering orthotropy.

After integrating by thickness the stresses at an arbitrary point of the i-thlayerwe move to linear forces, acting at the level of the middle surface of the same layer: $N_{1}^{(i)}, N_{2}^{(i)}$ are normal forces, $M_{1}^{(i)}, M_{2}^{(i)}$ are bending moments, $H^{(i)}$ is a torsion moment and $S^{(i)}$ are shear forces. Substituting the expression for forces by the expression for strains and after integrating we obtain elasticity relations: 


$$
\begin{aligned}
& N_{1}^{(i)}=B_{11}^{(i)} \varepsilon_{1}^{(i)}+B_{12}^{(i)} \varepsilon_{2}^{(i)}+C_{11}^{(i)} k 1+C_{21}^{(i)} k_{2}^{(i)} ; \\
& N_{2}^{(i)}=B_{21}^{(i)} \varepsilon_{1}^{(i)}+B_{22}^{(i)} \varepsilon_{2}^{(i)}+C_{21}^{(i)} k 1+C_{22}^{(i)} k_{2}^{(i)} ; \\
& M_{1}^{(i)}=C_{11}^{(i)} \varepsilon_{1}^{(i)}+C_{12}^{(i)} \varepsilon_{2}^{(i)}+D_{11}^{(i)} k 1+D_{21}^{(i)} k_{2}^{(i)} ; \\
& M_{2}^{(i)}=C_{21}^{(i)} \varepsilon_{1}^{(i)}+C_{22}^{(i)} \varepsilon_{2}^{(i)}+D_{21}^{(i)} k 1+D_{22}^{(i)} k_{2}^{(i)} ; \\
& H_{1}^{(i)}=2 D_{66}^{(i)} \chi_{12}^{(i)}+C_{66}^{(i)} \gamma_{12}^{(i)} ; S^{(i)}=B_{66}^{(i)} \gamma_{12}^{(i)}+2 D_{66}^{(i)} \chi_{12}^{(i)},
\end{aligned}
$$

Where rigidity characteristics $B_{11}^{(i)}, B_{12}^{(i)}, B_{22}^{(i)}, \ldots, D_{66}^{(i)}$ are defined by corresponding integral expressions.

To record shear stresses along the generatrix in the $i$-thjoint the following formulas are used [1]:

$$
\tau_{1}^{(i)}=\eta_{1}^{(i)} \Delta u^{(i)}, \tau_{2}^{(i)}=\eta_{2}^{(i)} \Delta v^{(i)},
$$

Where $\eta_{1}^{(i)}, \eta_{2}^{(i)}$ are the i-thjointrigidity factors; $\Delta u^{(i)}, \Delta v^{(i)}$ are the differences of longitudinal displacements of adjacent layers in the i-thjoint area in the direction of xand $\varphi$ coordinates respectively.

Differential equilibrium equations in displacements for the i-thlayer, given shear stresses, will be represented in accordance with the methodology $[1,3,4,5]$. The order of differential equations system determines the number of boundary conditions.

There have been regarded composite structures in the form of a circular cylindrical shell, closed in a transverse direction and bounded by two plane sections perpendicular to the axis of the cylinder. Boundary conditions have been specified on each of the two transverse layers along coordinate $X$. The periodicity conditions of the required function along coordinate $\varphi$ must be met.

In case of axisymmetric deformation of a composite cylindrical shell the system of differential equations is simplified [4]:

$$
\begin{gathered}
-D 11 \frac{d^{4} w}{d x}+\frac{d}{d x}\left(\sum_{i=1}^{I} C^{(i)} \eta^{(i)}\left(u^{(i+1)}-u^{(i)}+C^{(i)} \frac{d w}{d x}\right)\right)- \\
\frac{1}{r} \sum_{i=1}^{I+1}\left(B_{21}^{(i)} \frac{d u^{(i)}}{d x}+B_{22}^{(i)} \frac{w}{r}\right)+q^{n}=0 ; \\
B_{11}^{(i)} \frac{d^{2} u^{(i)}}{d x^{2}}+\frac{B_{12}^{(i)}}{r} \frac{d w}{d x}+\eta^{(i)}\left(u^{(i+1)}-u^{(i)}\right)-\eta^{(i-1)}\left(u_{\left.u^{(i)}-u^{(i-1)}\right)+}\right. \\
+\frac{d w}{d x}\left(\eta^{(i)} C^{(i)}-\eta^{(i-1)} C^{(i-1)}\right)=0,1 \leq i \leq I+1 .
\end{gathered}
$$

The equilibrium equations of a two-layer composite cylindrical shell under axisymmetric deformation will be represented as: 


$$
\begin{gathered}
-D 11 \frac{d^{4} w}{d x^{4}}+C^{2} \eta \frac{d^{2} w}{d x^{2}}-\left(B_{22}^{(1)}+B_{22}^{(2)}\right) \frac{w}{r^{2}}+\left(C \eta-\frac{B_{21}^{(2)}}{r}\right) \frac{d u^{(2)}}{d x}-\left(C \eta+\frac{B_{21}^{(1)}}{r}\right) \frac{d u^{(1)}}{d x}=-q_{3} ; \\
B_{11}^{(1)} \frac{d^{2} u^{(1)}}{d x^{2}}+\left(\frac{B_{12}^{(1)}}{r}+C \eta\right) \frac{d w}{d x}-\eta u^{(1)}+\eta u^{(2)}=0 \\
B_{11}^{(2)} \frac{d^{2} u^{(2)}}{d x^{2}}+\left(\frac{B_{12}^{(2)}}{r}-C \eta\right) \frac{d w}{d x}+\eta u^{(1)}-\eta u^{(2)}=0,
\end{gathered}
$$

Where $C$ is the distance between the layers middle surfaces. The boundary conditions for a composite structure with hinging ends (with $x=0 ; x=L$ ) are: $w=0 ; M 1=0, N_{1}^{(i)}=0, i=1,2$.

\section{Asymptotic representation of the solution}

The system of differential equations is solved by decomposition of required functions and the load vector into trigonometric series which, with given boundary conditions, has the following form:

$$
\begin{aligned}
& u^{(1)}(x)=\sum_{m=1}^{\infty} U_{m}^{(1)} \cos \left(\frac{m \pi x}{L}\right) ; u^{(2)}(x)=\sum_{m=1}^{\infty} U_{m}^{(2)} \cos \left(\frac{m \pi x}{L}\right) ; \\
& w(x)=\sum_{m=1}^{\infty} W m \sin \left(\frac{m \pi x}{L}\right) ; q_{3}(x)=\sum_{m=1}^{\infty} q_{m} \sin \left(\frac{m \pi x}{L}\right) .
\end{aligned}
$$

Under the evenly distributed load $q_{m}=\frac{4 q}{m \pi}$, where $\mathrm{m}=1,3,5$, etc. From the system of equilibrium equations of a two-layer composite cylindrical shell we move to the system of equations:

$$
\begin{aligned}
& D 11 \sum_{m=1}^{\infty} W m\left(\frac{m \pi}{L}\right)^{4} \sin \left(\frac{m \pi x}{L}\right)+C^{2} \eta \sum_{m=1}^{\infty} W m\left(\frac{m \pi}{L}\right)^{2} \sin \left(\frac{m \pi x}{L}\right)+ \\
& +\left(\frac{B_{22}^{(1)}+B_{22}^{(2)}}{r^{2}}\right) \sum_{m=1}^{\infty} W m \sin \left(\frac{m \pi}{L}\right)- \\
& \left(\frac{B_{21}^{(1)}}{r}+C \eta\right) \sum_{m=1}^{\infty} U_{m}^{(1)}\left(\frac{m \pi}{L}\right) \sin \left(\frac{m \pi x}{L}\right)+ \\
& +\left(C \eta-\frac{B_{21}^{(2)}}{r}\right) \sum_{m=1}^{\infty} U_{m}^{(2)}\left(\frac{m \pi}{L}\right) \sin \left(\frac{m \pi x}{L}\right)=\sum_{m=1}^{\infty} q_{m} \sin \left(\frac{m \pi x}{L}\right) .
\end{aligned}
$$




$$
\begin{aligned}
& \left(\frac{B_{12}^{(1)}}{r}+C \eta\right) \sum_{m=1}^{\infty} W m\left(\frac{m \pi}{L}\right) \cos \left(\frac{m \pi x}{L}\right)-B_{1}^{(1)} \sum_{m=1}^{\infty} U_{m}^{(1)}\left(\frac{m \pi}{L}\right)^{2} \cos \left(\frac{m \pi x}{L}\right)- \\
& -\eta \sum_{m=1}^{\infty} U_{m}^{(1)} \cos \left(\frac{m \pi x}{L}\right)+\eta \sum_{m=1}^{\infty} U_{m}^{(2)} \cos \left(\frac{m \pi x}{L}\right)=0 \\
& \left(\frac{B_{12}^{(2)}}{r}-C \eta\right) \sum_{m=1}^{\infty} W m\left(\frac{m \pi}{L}\right) \cos \left(\frac{m \pi x}{L}\right)-B_{1}^{(2)} \sum_{m=1}^{\infty} U_{m}^{(2)}\left(\frac{m \pi}{L}\right)^{2} \cos \left(\frac{m \pi x}{L}\right)+ \\
& +\eta \sum_{m=1}^{\infty} U_{m}^{(1)} \cos \left(\frac{m \pi x}{L}\right)-\eta \sum_{m=1}^{\infty} U_{m}^{(2)} \cos \left(\frac{m \pi x}{L}\right)=0 .
\end{aligned}
$$

The values of coefficients $W m, U_{m}^{(1)}, U_{m}^{(2)}$ of the required function are determined by the solution of a system of linear algebraic equations equating coefficients of the corresponding trigonometric functions.

\section{Computation}

To study the effect of interlayer braces rigidities on the stress - strain state of composite cylindrical shells under axisymmetric loading, computation of a two-layer cylindrical shell have been performed. The estimated facility is the reactor compartment of a nuclear power plant. The shell of the reactor compartment is in the form of a cylinder made of class C40 concrete. To ensure sealing of the shell, inner metal lining made of sheet carbon steel is provided. The lining is used for concreting as formworks and attached to concrete with anchors. Tightness tests are carried out under pressure of $0.05 \div 0.07 \mathrm{MPa}$. Maximum internal incidental pressure is $0.5 \mathrm{MPa}$. Before facility commissioning the shell is tested for tightness and strength with pressure of $0.575 \mathrm{MPa}$.

The shell parameters are as follows: length $L$ is $68 \mathrm{~m}$,internal diameter is $45 \mathrm{~m}$, thickness of the concrete layer $\mathrm{h}^{(1)}$ is $0.7 \mathrm{~m}$, thickness of the inner steel lining $\mathrm{h}^{(2)}$ is $12 \mathrm{~m}$. The boundary conditions correspond to hinging ends. The mechanical properties of isotropic layers are as follows: $E^{(1)}=2.4 * 10^{4} \mathrm{MPa}, v^{(1)}=0.2, E^{(2)}=2.1 * 10^{5} \mathrm{MPa}$, $v^{(2)}=0.3$. The joint rigidity is $\eta=0 \div 20 \mathrm{~N} / \mathrm{mm}^{3}$. The calculation is carried out for internal pressure with the intensity of $q=0.5 ; 1 \mathrm{MPa}$ under the evenly distributed load of $q=0.5 \mathrm{MPa}$ and localized load of $q=1 \mathrm{MPa}$.

\section{Results}

The computation of normal stresses on the external surfaces of the shell layers under the evenly distributed load of $q=0.5 \mathrm{MPa}$ and localized load of $q=1 \mathrm{MPa}$ has been 
accomplished. Normal stresses $\sigma_{1}^{(1)}$ of the first layer are calculated at $z^{(1)}=-\frac{h^{(1)}}{2}$, and values $\sigma_{1}^{(2)}$ of the second layer are calculated at $z^{(1)}=\frac{h^{(1)}}{2}$. The computation showed that a change in rigidity of the interlayer braces significantly affects the distribution of normal stress in the steel lining. Atanevenly distributed load and increasing joint rigidity $\eta=0 \div 20 \mathrm{~N} / \mathrm{mm}^{3}$ the maximum stresses in the first layer change 3.8 times, andover 2.5 times under the load localized in the central part. The values of joint rigidity $\eta$ exceeding

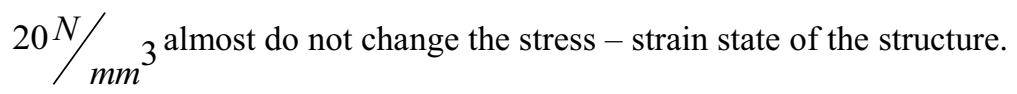

Figures 1 and 2 represent the graphs of distributions of deflections and bending moments in the first layer along the generatrix under localized load. According to the obtained results the changes of maximum deflections and bending moments at point $x=L / 2$ under localized load are $2.3 \%$ and $14 \%$, respectively. The maximum moments and deflections of an evenly loaded shell change by $12.7 \%$ and $1.7 \%$. In the computation of a shell subjected to internal pressure the near-anchorage area, where there are significant bending moments, ismostlystressed.

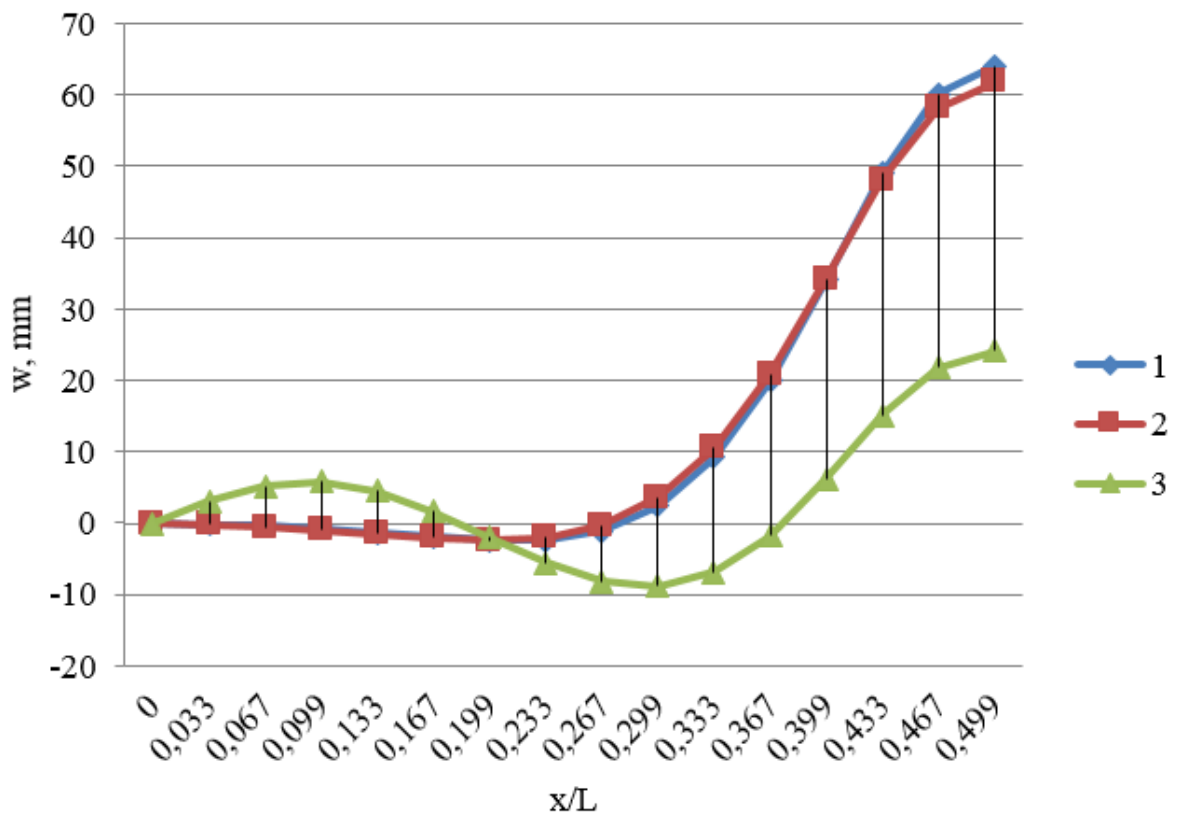

Fig. 1.Distribution of deflections in the first layer along the generatrix under localized load.Values of interlayer brace rigidities $1: 0.1 \mathrm{H} / \mathrm{mm}^{3} 2: 20 \mathrm{H} / \mathrm{mm}^{3}, 3: 10^{5} \mathrm{H} / \mathrm{mm}^{3}$. 


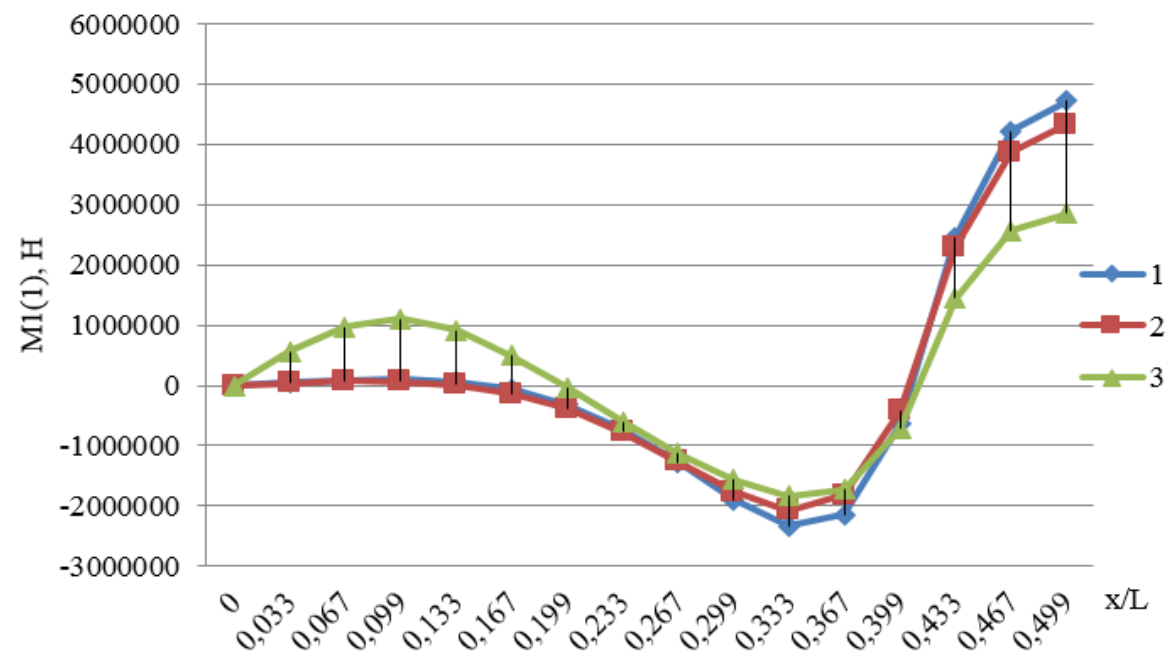

Fig. 2.Distribution of bending moments in the first layer along the generatrix under localized load. Values of interlayer brace rigidities 1: $0.1 \mathrm{H} / \mathrm{mm}^{3}, 2: 20^{\mathrm{H}} / \mathrm{mm}^{3}, 3:{ }_{10}^{5} \mathrm{H} / \mathrm{mm}^{3}$

\section{Conclusions}

The solution of the problem of bending of a closed two-layer cylindrical shell with steel layers under axisymmetric load showed that with rigidity exceeding ${ }_{10}^{5} \mathrm{H} / \mathrm{mm}^{3}$ the structure can be regarded as single, without taking into account slippage between layers. The variation of load distribution for a closed composite cylindrical shell showed that the interlayer brace rigidity under evenly distributed load significantly affects the stress - strain state at the near-anchorage area, and under local load at the central area. To assess the accuracy of the computation results exact solutions for a single-layer structure under axisymmetric loading (rigidity factors $\eta \rightarrow \infty, \eta \rightarrow 0$ ) are used. For example, if the interlayer brace rigidity of a two-layer shell is equal to zero, the bending rigidity is defined as the sum of rigidities of each individual layer. Provided that they coincide, i.e.the two layers are of the same thickness and material, we obtain a differential equation for cylindrical shell axisymmetric deformation [5]. The developed mathematical model in the form of differential equations allows studying the stress-strain state of composite cylindrical shells with different design parameters and load types.

\section{References}

1. A.R.Rzhanitsyn,Composite rods and plates(Stroyizdat, Moscow, 1986)

2. Y.E. Yakubovskiy,Nonlinear theory of bending and computation of composite plates and shallow shells with variable rigidity(Cand. Sci. (Eng.),Ekaterinburg, 1994)

3. V.V.Novozhilov, K.F. Chernykh, E.I. Mikhailovskiy,Linear theory of thin shells (Politekhnika, Moscow, 1991)

4. I.A. Donkova,Publishing House of Tyumen State University, 128 -133 (2013)

5. I.A. Donkova, Publishing House of Tyumen State University, 71 - 75 (2015)

6. Y. Yakubovskiy, V. Kolosov, B. Gulyaev, V. Goltsov, Procedia Engineering165, 1246-1253 (2016) 